\title{
Acciones de responsabilidad social universitaria en la oferta educativa del área económico-administrativa de la Universidad Veracruzana
}

\section{Actions of University Social Responsibility in the Educational Offer of the Economic-Administrative Area of the Universidad Veracruzana}

Kislev Dennys Nieto-Gassós'; Luis Alejandro Gazca-Herrera²

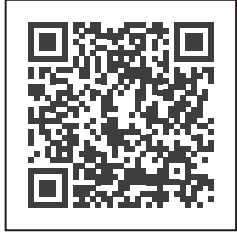

\section{Palabras clave:}

responsabilidad social, educación superior, fortalecimiento, gestión, buenas prácticas, escuelas de negocios.

Articulo de investigación:

Fecha de recepción:

29/04/2020

Fecha de aceptación: $14 / 08 / 2020$

Esta publicación se encuentra bajo licencia:

Creative Commons

Reconocimiento-

NoComercial-

SinObraDerivada 4.0

Internacional

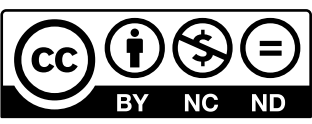

\section{Resumen}

La presente investigación destaca el tema de responsabilidad social universitaria como un elemento para desarrollar, establecer e implementar estructuras de gestión socialmente responsable. Es una investigación con alcance descriptivo basado en evidencia y en la experiencia, que recopila información de autores reconocidos del tema, entre los que destaca François Vallaeys. De igual modo, en función del compromiso social, se mencionan algunas de las múltiples acciones realizadas por la Universidad Veracruzana, en las cuales promueve la implementación de buenas prácticas dentro de una gestión responsable con calidad, contribuyendo así al desarrollo institucional de educación superior. Posteriormente se describen los elementos relacionados con el proceso de obtención al logro de distintivos de responsabilidad social universitaria, otorgados por la Asociación Nacional de Facultades y Escuelas de Contaduría y Administración,

1 Licenciada en Estadística. Maestra en Gestión de la Calidad, Universidad Veracruzana. Dirección general del Área Académica Económico-Administrativa, México.knieto@uv.mx, https://orcid.org/0000-0002-5118-1169

2 Licenciado en Sistemas Computacionales Administrativos. Maestro en Ciencias Administrativas y doctor en Administración Pública, Universidad Veracruzana, Facultad de Contaduría y Administración, México. Igazca@ uv.mx, ORCID: https://orcid.org/0000-0001-7637-2909 


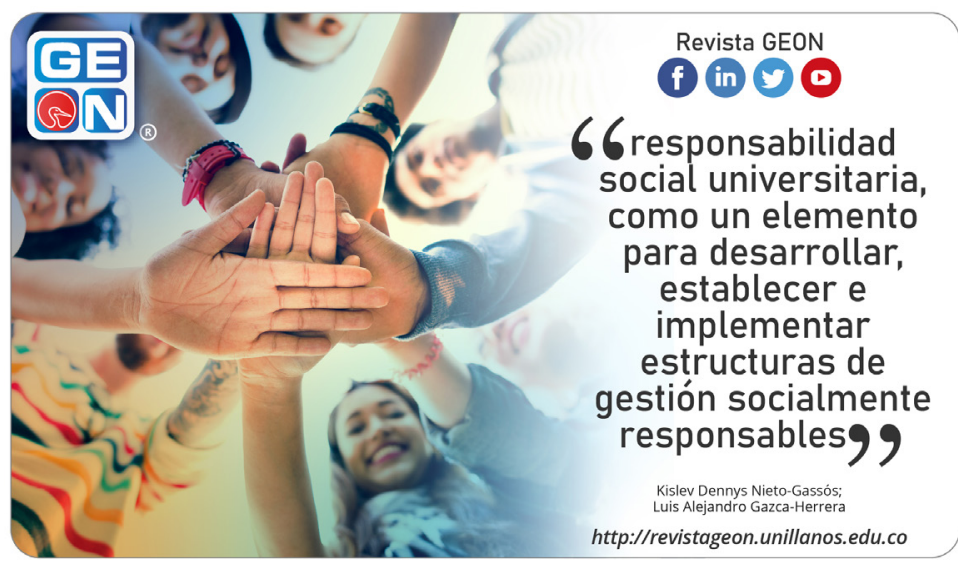

donde se destaca la participación de las facultades de negocios adscritas a la dirección general del área académica económico-administrativa de la Universidad Veracruzana, lo que favorece el fortalecimiento de la responsabilidad social, en el que, a partir de este referente, se perciben indicadores de evaluación, crecimiento y gestión de buenas prácticas en las entidades académicas para la toma de decisiones, para con ello propiciar, promover y coordinar un plan de mejora a partir del autoconocimiento para un impacto social.

Palabras clave: responsabilidad social, educación superior, fortalecimiento, gestión, buenas prácticas, escuelas de negocios.

Códigos JEL: M140

\section{Abstract}

This research highlights the issue of university social responsibility, as an element to develop, establish and implement socially responsible management structures. This is an investigation with descriptive scope based on evidence and experience, compiling and consulting information from recognized authors on the subject, highlighting François Vallaeys. In the same way, based on social commitment, some of the multiple actions carried out by the Universidad Veracruzana are mentioned, in which it promotes the implementation of good practices 
Cómo citar este artículo /

Toreference this article:

Nieto Gassos, K. D., \& Gazca Herrera, L. A. (2021). Accciones de Responsabilidad Social Universitaria en la oferta educativa del Área Económico-Administrativa de la Universidad Veracruzana. Revista GEON (Gestión, Organizaciones Y Negocios), 8(1). https://doi. org/10.22579/23463910.209 within responsible quality management, thus contributing to the institutional development of higher education; afterwards, elements related to the process of obtaining the achievement of university social responsibility marks, given by the Asociación Nacional de Facultades y Escuelas de Contaduría y Administración and schools of accounting and administration, where the participation of business faculties, attached to the general direction of the economic-administrative academy of the Universidad Veracruzana, enhancing the strengthening of social responsibility, in which, from this reference, indicators of evaluation, growth and management of good practices are perceived in academic entities for decision-making, and encourage, promote and coordinate an improvement plan based on self-knowledge for a social impact.

Keywords: Social responsibility, Higher education, Strengthening, Management, Good practices, Business schools.

\section{Introducción}

En la actualidad, para un mundo globalizado, la responsabilidad social universitaria representa el componente sustancial de las funciones de la academia, pues, como toda esencia social de los programas de formación, es y será una herramienta de obligatorio desarrollo, así como el establecimiento e implementación de estructuras de gestión para la toma de decisiones.

Desde este punto de vista, la Asociación Nacional de Facultades y Escuelas de Contaduría y Administración (ANFECA) otorga distintivos de responsabilidad social universitaria a través de la coordinación nacional de responsabilidad social a las escuelas de negocios afiliadas, que participan activamente en la incorporación de elementos para el fortalecimiento académico de las instrucciones de educación superior.

Bajo este escenario, particularmente la Dirección General del Área Académica Económico-Administrativa (DGAAEA), a través de la Universidad Veracruzana (UV), convoca para la formación académica a miles de estudiantes en diversas áreas de conocimiento. Esto demuestra el interés por fortalecer los temas de responsabilidad social universitaria, donde es central el trabajo colaborativo al 
interior y exterior de las entidades académicas, mediante el conocimiento de las necesidades de su entorno, así como la apropiación, creación e implementación de acciones con los grupos de interés, para un cambio de realidades existentes y diferentes en el estado de Veracruz.

\section{Contexto teórico}

Referenciando ampliamente las fuentes bibliográficas, en los últimos años, el tema de responsabilidad social (RS) ha tomado dimensiones en las cuales se identifica que las instituciones de educación superior (IES), a través del desarrollo de sus actividades universitarias establecidas en la misión y visión, se comprometen a un desarrollo ético institucional de calidad. Por consiguiente, la responsabilidad social universitaria (RSU) será una nueva forma de relación entre la universidad y la sociedad, desde una perspectiva de las partes interesadas. Es por ello por lo que es importante afianzar estos lazos para fortalecer el vínculo universidad-entorno social, en el que, según lo señalan Martínez, Rojas, Rodríguez y Carvallo (2008), la participación de la comunidad educativa universitaria y el logro de metas se deben dar en el marco de un ejercicio responsable, del juicio de los actos, el respeto a la libertad, la ética y la tolerancia, dentro de una perspectiva de compromiso y pertinencia institucional.

Siguiendo a François Vallaeys (2009), este postula a la RSU como la gestión justa y sostenible de los impactos sociales que genera la universidad en su actuar cotidiano, definidos en cuatro ámbitos: 1) organizacional; 2) educativo; 3) conocimiento; y 4) social. También destaca dos principales propósitos para la universidad: 1) la formación humana y profesional como propósito académico y 2) la construcción de nuevos conocimientos como propósito de investigación. Estos propósitos pueden ser vistos como oportunidades para generar nuevos productos o procesos y servir como instrumento diferenciador a partir de un propósito social.

Estos propósitos sociales implican procesos de cambio social, que involucran a las IES mediante aspectos relevantes de su misión institucional, en la cual está inmersa la RSU. Este es un esquema de análisis teórico de la RSU que propone Gaete (2012) desde la perspectiva del impacto que tiene el quehacer universitario en la sociedad.

Al mismo tiempo, Recalde, Cantero y Jara (2012) subrayan que la educación concibe que haya conocimiento, por lo que es transcendental que la universidad se relacione de manera coherente con teorías que sean prácticas para un crecimiento y fortalecimiento integral continuo en el desempeño de su labor en RSU, para la integración del individuo y de la familia a la sociedad. En el mismo sentido, Torres (2016), de acuerdo con Orozco y Martínez Baron (2012), afirma que las actividades de responsabilidad deben estar dirigidas al cambio de nuestros colaboradores para mejorar su calidad de vida y que esta se refleje en el mejoramiento de las condiciones de la familia y sus allegados. 
Siendo importante la RSU para la academia y su entorno, es relevante la inclusión en la formación de los futuros profesionales. Ramírez, Moctezuma y González (2017) afirman que se requiere mayor interés e involucramiento de los profesores para promover en sus alumnos la vocación social, de tal manera que el aula sea un espacio de apertura social, explotación de habilidades y apertura a nuevos saberes, donde el estudiante fomente la solidaridad, el liderazgo, el diálogo, la formulación de la crítica constructiva, el análisis objetivo y la cooperación. Aunado a esta formación académica, se ha comenzado a relacionar las gestiones internas de las entidades académicas, por medio de la ANFECA, que define a la RSU como un compromiso para promover la calidad y la ética del desempeño de las escuelas y facultades de negocios mediante una gestión responsable de los impactos educativos, en la generación y aplicación del conocimiento, organizacional, ambiental y social que sus instituciones afiliadas adoptan para buscar el desarrollo humano sostenible. Así mismo, precisa el concepto de buenas prácticas para referirse a las formas óptimas de ejecutar un proceso que sirve de referente para otras instituciones, las cuales deben ir acompañadas de indicadores para asegurar una buena gestión y permitir a los responsables institucionales decidir si serán necesarios componentes adicionales o correcciones de rumbo para lograr sus objetivos, y lo plantea como la herramienta para hacer tangible a la RSU (ANFECA, 2015).

\section{Materiales y métodos}

La justificación de la presente investigación se fundamenta, ante todo, en que la RSU revela el funcionamiento y la orientación al auxilio de las necesidades y problemas existentes de la sociedad local, regional, estatal y de un país. Por consiguiente, las IES están obligadas a ejercer con responsabilidad social las acciones y decisiones en relación con las funciones de docencia, investigación, extensión y gestión, de acuerdo con las necesidades sociales, para crear un vínculo responsable entre universidad-sociedad-empresa-gobierno y garantizar así el bien sustentable. Por estas razones, en particular la DGAAEA de la UV cuenta con programas educativos de licenciatura de Contaduría y Administración en distintas entidades académicas de las cinco regiones del estado de Veracruz, las cuales apuestan a la obtención de un distintivo como elemento para el fortalecimiento de las buenas prácticas, promovidas con ética y calidad para formar ciudadanos responsables con su entorno.

Por lo expresado con anterioridad, la problemática surge con la intención de conocer y profundizar el tema de responsabilidad social universitaria mediante los reconocimientos que avalúan las buenas prácticas de los programas educativos de las entidades académicas adscritas a la DGAAEA de la UV.

Es por ello por lo que el objetivo de la investigación fue describir a la RSU como la apertura de las buenas prác- 
ticas, lo que es un elemento característico para las facultades adscritas a la DGAAEA y hace tangible su compromiso por promover la calidad y la ética en el desempeño de una gestión responsable.

En este sentido, la UV, por tratarse de una institución pública de educación superior y autónoma de interés social del estado de Veracruz, se ha caracterizado por su compromiso real con la sociedad, fomentando y contribuyendo a este compromiso desde la academia. En relación con esto, se muestran algunas acciones generadas por la UV para formalizar una identidad de RSU, que busca promover al interior de la comunidad universitaria la capacitación, los talleres y los coloquios orientados al cambio sistémico mediante el establecimiento de la política y el concepto de RSU UV, así como los alcances y objetivos mediatos de líneas de acción concretas, para en el futuro dar a conocer información sobre la calidad de los resultados sobre la pertinencia académica y los beneficios del quehacer como universidad enmarcados en la responsabilidad social y la ética.

En la figura 1 se muestra la línea de tiempo de las actividades realizadas al interior de la universidad en cuanto a la RSU UV.

Bajo este escenario, la UV es parte fundamental en la sociedad veracruzana, en el entendido que su misión posee un impacto social, regional, nacional e internacional, puesto que, constantemente, trabaja en el fortalecimiento de una universidad socialmente responsable, mediante sus programas y la generación de líneas de acción y metas por alcanzar. Las anteriores afirmaciones nos Ilevan

Figura 1. Línea de tiempo de las actividades realizadas al interior de la UV para la RSU.

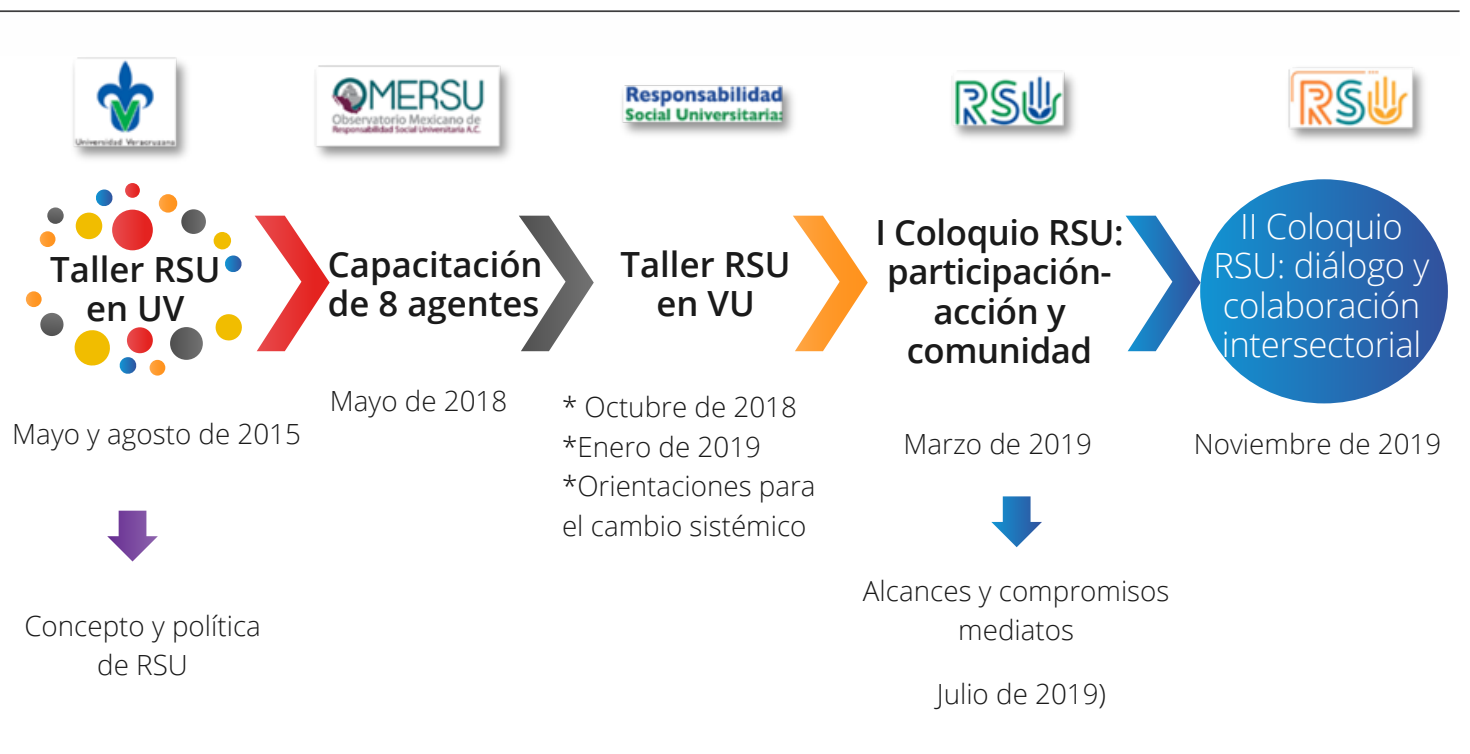

Fuente: elaboración propia de los autores. 
como DGAAEA a interiorizar y conocer los distintivos de RSU otorgados por la ANFECA como un elemento que apoya el fortalecimiento de la RSU, que es un instrumento de suma importancia que da cumplimiento a las acciones para las buenas prácticas en las IES.

\section{Resultados y discusión}

La coordinación nacional de RSU de la ANFECA presenta un modelo de RSU como elemento diferenciador incorporado a la cultura institucional y al sistema de gestión de los programas educativos en las IES, fundamentado a partir de las expectativas de los grupos de interés Ilamados Stake-holders externos (otros organismos, medios, gobierno, preparatorias, padres de familia y prospectos) y los Stake-holders internos (docentes, funcionarios y administradores, alumnos, egresados, empresas y reclutadores) en el desempeño de los principios y materias fundamentales relacionados con la operación de las IES.

Teniendo en cuenta este modelo y desde la perspectiva de las buenas prácticas, las IES afiliadas a la ANFECA tramitan, con base en la convocatoria del distintivo RSU, su compromiso por promover la calidad y la ética en el desempeño a favor de una gestión responsable en cinco ejes de impacto, definidos como: 1) educación; 2) generación y aplicación del conocimiento; 3) organización; 4) social; y 5) ambiental; además de seis principios básicos, a saber: 1) compromiso ético; 2) transparencia; 3) rendición de cuentas y no corrupción; 4) respeto a los derechos humanos; 5) cultura de la legalidad y respeto a las normas; y, por último, 6) participación ciudadana y sustentabilidad. Asimismo, todas aquellas instituciones de negocios afiliadas por primera vez y las partícipes de refrendar el distintivo de RSU deben cumplir con lo dispuesto en la guía, remitiendo a: a) formato de solicitud; b) autodiagnóstico: c) plan de mejora: y d) evidencias que enmarcan dicha convocatoria.

A continuación, se muestra el diagrama de proceso para la obtención del distintivo RSU, en el que se asigna un comité de tres pares dictaminadores que efectúan la revisión de la información proporcionada por la institución; si dicha evaluación es aprobada, se emite una resolución favorable para que se otorgue el distintivo de RSU (véase figura 2).

En caso de que el comité dictaminador pida información adicional a la institución solicitante, esta deberá atender los requerimientos anexos que pidan los pares, con el fin de puntualizar la obtención del distintivo RSU y permitir una resolución favorable. De esta forma, el dictamen de valoración que emiten los pares se presenta en tres tipos: a) aprobado sin observaciones; b) aprobado con observaciones; y c) negativo. Dicho distintivo tiene una vigencia de tres años a partir de la entrega del dictamen en la celebración de la asamblea nacional de la ANFECA. Las instituciones beneficiadas con el distintivo RSU deben presentar de manera anual a la coordinación nacional de RSU y al coordinador regional de su zona un 
Figura 2. Diagrama del proceso para la obtención del distintivo RSU.

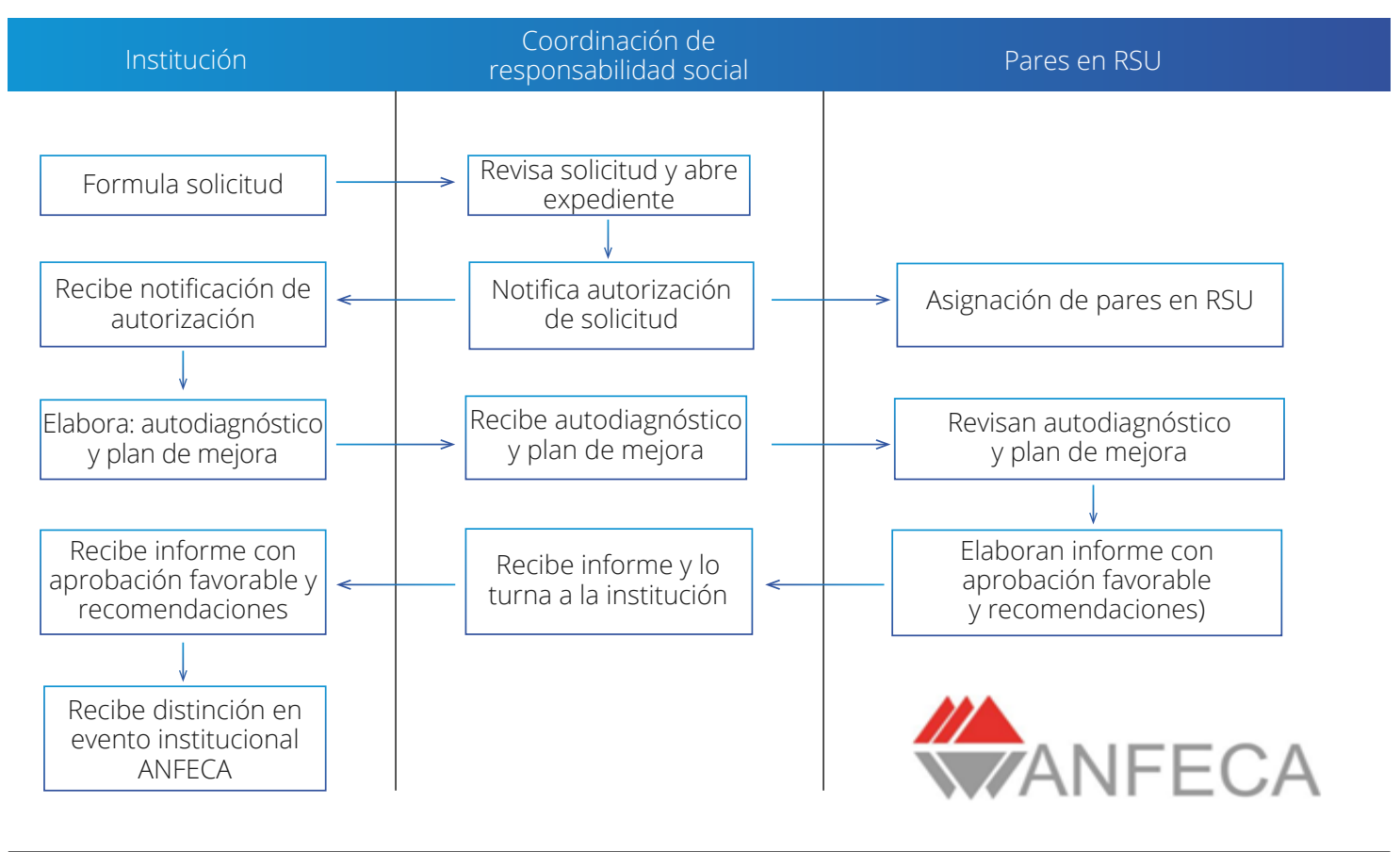

Fuente: adaptada de Universidad Veracruzana (2017).

reporte de avance del plan de mejora de las recomendaciones recibidas, así como las buenas prácticas implementadas en su institución.

Por otra parte, cabe señalar que la función del par evaluador es determinar, sea valorada por parte de la IES, la formación de la cultura de RSU interna y de los avances significativos de RSU respecto al modelo ANFECA, por lo que la revisión del instrumento consiste en que:

1. La IES se autoevalúa en el apartado de datos generales, conformado por 11 indicadores, argumentando y presentando un plan de mejora por cada indicador y valorando los compromisos de las buenas prácticas.
2. La IES evalúa los elementos del apartado de autodiagnóstico para cada eje de impacto y de cada principio, en el cual, por medio de prácticas reales, se tiene un referente y una evidencia de cada indicador; asimismo se debe de argumentar y presentar un plan de mejora por cada indicador.

De esta manera, los pares dictaminadores asignan a cada uno de los ejes una escala de medición para evaluar las prácticas reales y establecen un puntaje de cumplimiento en una escala ideal de 1000 puntos (véase tabla 1).

Lo mismo ocurre para el apartado de datos generales, donde los pares dictaminadores ANFECA asignan una ponderación de puntaje máximo idóneo 
Tabla 1.Escala de dimensiones por ejes de impacto y principios RSU ANFECA

\begin{tabular}{|c|c|c|c|c|c|}
\hline Ejes de impacto & Principios & $\begin{array}{c}\text { Total de } \\
\text { indicadores } \\
\text { de prácticas } \\
\text { reales }\end{array}$ & $\begin{array}{c}\text { Total de } \\
\text { puntos }\end{array}$ & $\begin{array}{c}\text { Total de } \\
\text { escala }\end{array}$ & $\%$ \\
\hline 1. Educativo & \multirow{6}{*}{$\begin{array}{l}\text { 1. Compromiso ético } \\
\text { 2. Transparencia, rendición de } \\
\text { cuentas y no corrupción } \\
\text { 3. Respeto a los derechos humanos } \\
\text { 4. Cultura de la legalidad y respeto a } \\
\text { las normas } \\
\text { 5. Participación ciudadana } \\
\text { 6. Sustentabilidad }\end{array}$} & 24 & 75 & 300 & 0,3 \\
\hline $\begin{array}{l}\text { 2. Generación } \\
\text { y aplicación del } \\
\text { conocimiento }\end{array}$ & & 8 & 75 & 100 & 0,1 \\
\hline 3. Organizacional & & 31 & 75 & 387,50 & 0,3875 \\
\hline 4. Social & & 9 & 75 & 112,50 & 0,1125 \\
\hline 5. Ambiental & & 8 & 75 & 100 & 0,1 \\
\hline Total & & 80 & 375 & 1000 & 1 \\
\hline
\end{tabular}

Fuente: elaboración propia de los autores.

de 275 puntos para el cumplimento del $100 \%$ de los indicadores. De esta manera, una vez evaluada la incorporación de herramientas de gestión en las IES, el equipo dictaminador obtiene la suma de las dimensiones de ambos apartados (datos generales y el autodiagnóstico), estableciendo un puntaje máximo para una IES, un total de 1275 puntos o bien un puntaje mínimo de 765 puntos para la obtención del reconocimiento RSU ANFECA.

Dentro de esta apertura, la DGAAEA en sus atribuciones se ha caracterizado por orientar y contribuir al logro de objetivos desde la academia. Por tanto, a través de las facultades de Contaduría y Administración adscritas a esta dirección general de la UV, las entidades académicas de la DGAAEA se han sometido a evaluación mediante instrumentos RSU ANFECA, lo que las ha hecho acreedoras de las buenas prácticas, desde el año 2016, con el primer otorgamiento del distintivo y su respectivo refrendo en el 2019, en el cumplimiento de principios fundamentales de RSU, promoviendo la calidad y ética del desempeño en las entidades académicas, mediante una gestión responsable en el impacto educativo (véase tabla 2).

Del mismo modo, el sistema de enseñanza abierta de la UV se suma obteniendo el distintivo RSU en el año 2019 para las facultades de Contaduría y Administración adscritas a la DGAAEA de las regiones de Xalapa, Veracruz, Córdoba-Orizaba (campus Ixtaczoquitlán), Poza Rica-Tuxpan y Coatzacoalcos.

En estos escenarios, la DGAAEA, desde la experiencia, identificó que, al obtener el distintivo RSU de ANFECA, las facultades no cuentan con un documento oficial de retroalimentación para el seguimiento de las recomendaciones de los ejes y principios que emite el par dictaminador. A causa de esta deficiencia, se frena una parte primordial para el fortalecimiento de 
Tabla 2. Distintivos RSU ANFECA a las entidades académicas de la DGAAEA

\begin{tabular}{ll|cc}
\multicolumn{1}{c|}{ Región } & \multicolumn{1}{c|}{ Facultad } & $\begin{array}{c}\text { Distintivo } \\
\mathbf{2 0 1 6}\end{array}$ & $\begin{array}{c}\text { Distintivo } \\
\text { Refrendo 2019 }\end{array}$ \\
\hline Xalapa & Facultad de Contaduría y Administración & $*$ & $*$ \\
\hline Xalapa & Facultad de Ciencias Administrativas y Sociales & $*$ & $*$ \\
\hline Veracruz & Facultad de Contaduría & $*$ & $*$ \\
\hline Veracruz & Facultad de Administración & $*$ & $*$ \\
\hline Ixtaczoquitlán & Facultad de Contaduría y Administración & $*$ & $*$ \\
\hline Tuxpan & Facultad de Contaduría & $*$ & $*$
\end{tabular}

Fuente: elaboración propia con base en datos de http://www.anfeca.unam.mx/coordinaciones_responsabilidad.php

la RSU deseable al interior de las entidades académicas, donde las líneas de acción son importantes para transitar de las buenas intenciones a las acciones concretas.

10

Aunado a la importancia de la responsabilidad social en la academia, podemos expresar que no es suficiente conservar un distintivo de RSU; sin embargo, participar en y conocer el instrumento de evaluación ANFECA sirve de referente para visualizar los elementos que apoyan directamente a la RSU o para saber qué orientación habrá que darles para que sí funcionen y con ello establecer e implementar estructuras de gestión para la toma de decisiones que auxilien a la solución de problemas sociales. Por tanto, la DGAAEA, en el cumplimiento y la aplicación de las políticas, programas y disposiciones que regulan su funcionamiento, pretende realizar acciones de forma gradual en los años 2020 y 2021, por medio de un análisis de reporte de los programas educativos de las facultades, para manifestar si al interior de cada unidad académica adscrita a esta dirección se tienen procesos de implementación, promoción, fortalecimiento o iniciativas de responsabilidad social, con herramientas adecuadas relacionadas con la gestión, vinculación, proyectos, servicios, convenios y programas efectivos.

\section{Conclusiones}

En conclusión, la responsabilidad social universitaria y los modelos que llevan a los distintivos de responsabilidad social permiten identificar diferentes aspectos relevantes en la toma de decisiones y el trabajo colaborativo en los procesos de implementación y fortalecimiento de la RSU, para plasmar las oportunidades de mejora a partir del vínculo universidad-entorno social.

Esto indica que se puede precisar condiciones hacia contribuciones futuras en la generación de estructuras de mejora a partir de indicadores de crecimiento y gestión de buenas prácticas para la toma de decisiones.

En lo argumentando, la DGAAEA necesita autoconocerse y afirmarse en 
las fortalezas y las limitaciones en cuanto a las capacidades de enseñanza-aprendizaje con respecto a la RS. Esto le permitirá autovalorar los programas educativos de excelencia en el estado de Veracruz e identificar las alianzas con las empresas y el gobierno y aumentar así la comunicación institucional de los compromisos conjuntos para conocer, difundir y transmitir lo que se está haciendo en materia de RS, para la pertinencia e impacto social en la formación integral del estudiantado y del profesorado de la máxima casa de estudio, con el fin de desarrollar, establecer e implementar estructuras de gestión socialmente responsable.

Esto apoya la teoría mencionada al inicio de esta investigación con la que se concuerda y también se busca lo propuesto por Vallaeys (s/f), que afirma que para el mejoramiento de las capacidades del alumnado en lo social y educativo de un objetivo de RSU idóneo debe darse la capacitación de la comunidad universitaria, así como la enseñanza basada en proyectos con impacto social para la orientación hacia la solución de problemas sociales y las necesidades del saber y del mundo actual.

\section{Referencias}

Asociación Nacional de Facultades y Escuelas de Contaduría y Administración (ANFECA). (2013). Distintivos de responsabilidad social universitaria http:// www.anfeca.unam.mx/coordinaciones_responsabilidad.php

Fernández, C., Delpiano, C., \& Ferari, J. M. (2006). Responsabilidad social universi- taria - Una manera de ser universidad. Teoría y práctica en la experiencia chilena. Proyecto Universidad: Construye País. https://bit.ly/2ErCBFB

Gaete-Quezada, R. A. (2012). Responsabilidad social universitaria: una nueva mirada a la relación de la universidad con la sociedad desde la perspectiva de las partes interesadas. Un estudio de caso [tesis de doctorado, Universidad de Valladolid]. Repositorio Documental UVADOC. http://uvadoc.uva. es/handle/10324/923

Modelo de responsabilidad social universitaria de la asociación nacional de facultades de contaduría y administración. ANFECA (2017). https://www. uv.mx/fca/files/2016/11/Distintivo-RSU-ANFECA-2017.pdf

Martínez de Carrasquero, C., Mavarez, R., Rojas, L., \& Carvallo, B. (2008). La responsabilidad social universitaria como estrategia de vinculación con su entorno social. Frónesis, 15(3), 81-103. https://bit.ly/3gooiiq

Ninco-Hernández, F., \& Duque-Orozco, Y. (2017). Enfoques de la responsabilidad social empresarial innovadora: alternativas para las organizaciones de hoy. Revista Gestión, Organizaciones y Negocios, 4(1), 162-175. https://doi. org/10.22579/23463910.52

Observatorio Mexicano de Responsabilidad Social Universitaria (OMRSU A.C.) (2014). https://www.responsabilidadsocial.net/observatorio-mexicano-de-responsabilidad-social-universitaria-omersu/

Organismo Internacional de Estandarización. (2010). Guía de responsabilidad Social ISO 26000: 2010 (es). Online Browsing Platform (OBP). https://www.iso.org/obp/ui\#iso:std:iso:26000:ed-1:v1:es 
Pérez Alayón Juan de Dios, Vallaeys François (2016). Prácticas y Modelos de Responsabilidad Social Universitaria en México. Proceso de transformación de la universidad. Primera edición, Mexico, D.F. http://publicaciones.anuies.mx/pdfs/libros/Libro221.pdf

Ramírez-Vázquez, Y., Moctezuma-Merlo, P., \& González-Muñoz, O. (2017). Importancia de la responsabilidad social en la formación de los futuros profesionales. Instituto de Investigaciones y Estudios Superiores de las Ciencias Administrativas, 53-65. https://www.uv.mx/ iiesca/files/2017/10/07CA201701.pdf

Recalde, H., Cantero, W., \& Jara, J. (2012). Importancia de la responsabilidad social universitaria como desarrollo Social de la ciudad de Coronel Oviedo. Revista Gestão Universitaria na América Latina, 5(4), 267-280. DOl: http://dx. doi.org/10.5007/1983-4535.2012v 5n4p266

Torres-Flórez, D. (2016). La responsabilidad social empresarial, más allá de lo legal. Revista Gestión, Organizaciones y Negocios, 3(1), 4-4. https://doi. org/10.22579/23463910.66

Universidad Veracruzana. (2017). Distintivo RSU ANFECA. Asamblea Regional ANFECA. https://www.uv.mx/fca/ files/2016/11/Distintivo-RSU-ANFECA-2017.pdf
Universidad Veracruzana. (2019, 28 y 29 de noviembre). I/ Coloquio Responsabilidad social universitaria: diálogo y colaboración intersectorial. Responsabilidad Social Universitaria. https://bit. ly/34sdWeU

Universidad Veracruzana. (2019). Concepto de RSU UV. Responsabilidad Social Universitaria. https://bit.ly/31 nAwUk

Universidad Veracruzana (2019). Política de RSU UV. Responsabilidad Social Universitaria. https://bit.ly/3j5TEvY

Vallaeys, F. (2014). La responsabilidad social universitaria: un nuevo modelo universitario contra la mercantilización. Revista Iberoamericana de Educación Superior, 5(12), 105-117. http://www.scielo.org.mx/pdf/ries/ v5n12/v5n12a6.pdf

Vallaeys, F., De la Cruz C., \& Sasia, P. M. (2009). Responsabilidad social universitaria: Manual de primeros pasos. McGraw-Hill Interamericana Editores.

Vallaeys, F., \& Pérez-Alayón J. D. (2016). Prácticas y modelos de responsabilidad social universitaria en México. Proceso de transformación en la universidad. Asociación Nacional de Universidades e Instituciones de Educación Superior. http://publicaciones.anuies. mx/ pdfs/libros/Libro221.pdf 